cial [1]. One of the possible causes of digestive tract diseases in children is the failure in the constantly growing body of regulatory systems and, above all, the autonomic nervous system [2]. Violations of vegetative homeostasis, leading to the disruption of the functions of organs and systems, contribute to a change in the level of metabolism and energy in the body and are an important pathogenetic link of many chronic diseases, including gastrointestinal ones[3]. It is assumed that the identification of features of the functioning of the autonomic nervous system and heart rate parameters will allow timely not only to diagnose, but also to correct their deviations [4].

The purpose of this study was to explore the features of changes in cardiac rhythm and conductivity, identified on an electrocardiogram (ECG), in children with chronic gastroduodenal pathology.

Materials and methods: The main group (I) consisted of 41 children aged 4 to 10 years (mean age 8,2) with chronic pathology of the gastroduodenal zone. Gender distribution was as follows: 18 boys (43,9\%) and 23 girls - (56,1\%). The control group (II) was 45 conditionally healthy children (without pathology of the gastrointestinal tract; from them - 18 girls (40\%) and 27 boys (60\%), the average age $-8,6$ years, groups were comparable by age and gender. The diagnosis was established on the basis of complaints, anamnesis of the disease, general clinical examination, data of fibroadastroduodenoscopy, ultrasound examination of the abdominal cavity organs, tests for the presence of Helicobacter pylori.

Results and discussion: The duration of the disease in the main group was as follows: 7 gastroduodenal pathology $(17,1 \%)$, duration of the disease 1 year - 14 people $(34,1 \%)$, duration of the disease over 1 year - 20 people $(48,8 \%)$; the average duration of the disease in children was 2,2 years. Diseases of the stomach and duodenum (gastritis, gastroduodenitis) predominated in the study group - 25 children (60,9\%), from them - 61,5\% was diagnosed erythematous gastritis, in $7 \%$ children - gastroesophageal reflux; diseases of the gallbladder and biliary tract (dysfunction of the biliary tract) - 3 patients (7,3\%); pancreas diseases (manifestations of dispensiveness, pancreatopathy) - 13 children (31,7\%). 39\% of children in first group had concomitant pathology: weight loss in 12,5\%. 1/3 children form this group had concomitant pathology: weight deficit was in 12,5\%, cardiac pathology - in 37,5\%, orthopedic pathology - in $18,7 \%$ and $14 \%$ of children had endocrine and neurological diseases. According to the results of ultrasound in the main group, changes in the pancreas (structural changes, densification of the Virsung duct) were found in $41,2 \%$ patients, changes in the liver (reactive changes in the liver parenchyma, compaction of the periportal structures) were detected in $32,3 \%$ and changes in the gallbladder (deformation, inflection) - in $26,5 \%$ children.

Having studied ECG data in the main group of changes, 95,1\% of children were registered, which is significantly more likely than in children of the control group 48,8\% ( $p<0.05)$. So the frequency of bradycardia was $53,6 \%$, which is significantly more likely than in group II $(20 \%)(p<0,05)$, which have indicated the vagal nature of these diseases. Ectopic rhythm and rhythm migration also occurred more often in patients of the first group, but without significant differences $(19,5 \%$ и $8,9 \%$ accordingly). Normocardia prevailed in the children of the control group (70\%) (p $<0,01)$. Violations of the conduction in the ventricles were significantly more frequent in children and adolescents with gastroduodenal pathology $(65,9 \%)$ than in the control group $(24.4 \%)(p<0,05)$. On average, one patient of the first group had 1,2 $\pm 0,1$ rhythm and conduction disorders and in $I I-0,6 \pm 0,2$ (2 times less) ( $p<0.05)$. In addition, the combination of arrhythmias and conduction disorders was noted only in the children of the main group (15,9\%).

Conclusions: Thus, ECG analysis indicates significant differences in the quantitative and qualitative nature of electrogenesis in children and adolescents in the main group. This allows us to distinguish a group of changes (in the form of bradycadias and conduction disorders) associated with the pathology of the gastrointestinal tract and the peculiarity of the functioning of regulatory systems. The high frequency of cardiac arrhythmias and conduction in gastroduodenal pathology indicates a considerable significance of these disorders in the genesis of the development of these conditions. When carrying out medical and recreational activities in children with chronic gastroduodenal diseases, along with standard methods of examination, it is necessary to study the condicion of the autonomic nervous system for more effective pathogenetic and symptomatic treatment.

\title{
References:
}

1. Suprun I.M., Makarova V.I. State of the autonomic nervous system in children with atopic dermatitis, with chronic diseases of the stomach and duodenum // Human Ecology - 2006. - № 12. - P. 22-26.

2. Sikorsky A.V. Symptomatic arterial hypotension in children with chronic gastroduodenal pathology: peculiarities of central hemodynamics and heart rhythm // Journal of GGMU. - 2003 - № 1. - P. 28-31.

3. Zanko S.N. Variability of heart rhythm and cerebral hemodynamics in children with chronic gastroduodenal pathology // Reviewed scientific and practical journal "Protection of motherhood and childhood". - 2005. - №1(6). - P. 17-23.

4. Chupak E.L. Features of vegetative reactivity in adolescents with bronchial asthma, taking into account the initial vegetative tone // Proceedings of the VII Congress of pulmonologists in Siberia and the Far East (with international participation).- Blagoveshchensk, 2017. P. 207-210.

UDC 616.24-002-005:616-008.9 DOI 10.22448/AMJ.2017.3.143-146

\section{PECULIARITIES OF CLINIC AND HEMODYNAMICS IN COMORBID COURSE OF OBSTRUCTIVE PULMONARY DISEASE AND METABOLIC SYNDROME}

\author{
O.A. Tanchenko, S.V. Naryshkina
}

FSBEI HE Amur State Medical Academy

Summary: Chronic obstructive pulmonary disease (COPD) belongs to a group of chronic inflammatory lung diseases characterized by a partially reversible airflow limitation. Among the numerous comorbid conditions of COPD, in more detail we examined the role of factors of systemic inflammation, arterial stiffness, carbohydrate and lipid metabolism in the progression of the comorbid course of COPD and the main components of the metabolic syndrome. It was revealed that an increase of arterial stiffness and systemic inflammation appear to be an indepen- 
dent risk factor of cardiovascular complications and the progression of cardiovascular pathology with the comorbidity of COPD and metabolic syndrome. Early detection of changes in arterial stiffness and metabolic disorders will allow correcting the therapy and preventing adverse consequences of hemodynamic complications in proper time.

Key words: chronic obstructive pulmonary disease, metabolic syndrome, arterial stiffness.

At the present time, there is a high prevalence of COPD in individuals older than 40 years $[3,7]$. Case fatality rate of COPD patients reaches $28 \%$ in the population of patients aged 65 years and older [2, 8]. In patients with frequent COPD exacerbations, there is an increase in the mortality rate from the cardiovascular diseases, which makes it possible to suggest the important role of vascular catastrophe in the pathogenesis of fatal exacerbations of COPD [6]. In a number of clinical studies, proper attention is paid to the increasing rate of pulmonary artery thromboembolism among the causes of COPD mortality, reaching 29\% [7]. At the present time, among the numerous comorbid conditions of COPD, cardiovascular diseases, metabolic syndrome and diabetes mellitus are under consideraton [2].

The prevalence of metabolic syndrome, including abdominal obesity, arterial hypertension, dyslipidemia, carbohydrate metabolism disorder in patients with COPD, is 21-53\%. An increase in the incidence of metabolic syndrome among COPD patients may be associated with an increase in the prevalence of obesity, a decrease in physical activity, smoking, the use of glucocorticosteroids, and the progression of insulin resistance $[1,8]$.

According to different authors, the frequency of combination of diabetes mellitus (DM) with COPD varies from 2 to $16 \%$. It is interesting to note that in the development mechanisms of COPD and type $2 \mathrm{DM}$, there are many common mechanisms. For instance, both in type 2 diabetes mellitus and in COPD, there is a decrease in the phagocytic function of neutrophils and insufficiency of the T cell link of immune system [4, 5]. It has been established that the main feature of the hypercoagulability syndrome is the comorbidity of COPD with abdominal obesity, which occurs in patients with obstructive pulmonary disease in $31 \%$ of cases $[1,6]$. Thus, the study of the mechanisms of interaction of systemic inflammatory reactions, the condition of haemodynamics of great vessels associated with COPD and metabolic syndrome will improve the methods of prevention and treatment of complications of this comorbid pathology. In the available literature there are no clinical studies performed by means of systematic approach to assess the effect of metabolic syndrome components on the clinico-instrumental characteristics of COPD patients.

The aim of the research: to study the effect of metabolic syndrome components on changes in cytokine status, arterial stiffness in patients with COPD and to evaluate their relationship with endothelin-1, leptin, insulin resistance, glycated hemoglobin, lipid spectrum, anthropometric measures. Materials and methods: The complex study included 94 patients aged from 38 to 69 years with the moderate stage of COPD. Detection of COPD, allocation of its stages and its severity was carried out according to the international program "Global Initiative for COPD 2016". Evaluation of the severity of dyspnea was carried out according to the scale of the Medical Research Council Scale. Diagnosis of the metabolic syndrome was established based on criteria developed by the International Federation of Diabetes of 2014 [1]. The body mass index (BMI) in patients was $36.31 \pm 2.71 \mathrm{~kg} /$ $\mathrm{m} 2$. The waist circumference in men was on the average $107.3 \pm 6.14 \mathrm{~cm}$, in women $104.8 \pm 5.12 \mathrm{~cm}$. The waist-to-hip ratio in men was $1.11 \pm 0.06$, in women $1.07 \pm 0.04$. Obesity of the 1 st degree was diagnosed in 31 patients (39.7\%), 2nd degree - in $28(35.9 \%)$, 3rd one - in 19 (24.4\%). COPD patients were assigned to groups B and C according to the classification of the risk of adverse outcomes and comorbid course and they were divided into 3 groups: the 1st group consisted of 28 patients with isolated form of COPD, the 2 nd group included 34 COPD patients with hypertensive form of metabolic syndrome, the 3rd group - 32 patients with COPD in combination with a diabetic variant of metabolic syndrome. The control group included 23 healthy individuals (12 men and 11 women) with a BMI from 18.5 to 24.9 $\mathrm{kg} / \mathrm{m} 2$, comparable to the study group by age and gender characteristics. The level of endothelin-1 was determined by high-sensitivity enzymoimmunoassay with the use of BIOMERICA test systems (Australia). The study of content of the cytokines level - interleukin (IL)-4, IL-6, IL-8, IL-10, tumor necrosis factor-alpha (TNF- $\alpha$ ), vascular endothelial growth factor was carried out with the help of test systems of Vector-Best CJSC by the method of enzyme immunoassay. The study of the C-reactive protein (CRP) was conducted by means of sets for the enzyme-linked immunosorbent assay with high sensitivity of "BIOMERICA" company. Oxygen saturation level (SpO2) was estimated using a MD300C pulse oximeter (Little Doctor, Singapore). For the purpose of studying exercise tolerance, a six-minute step test was performed. Insulin resistance was determined via calculation of insulin resistance index on the HOMA (Homeostasis Model Assessment) criterion - [fasting insulin $\mathrm{mcU} / \mathrm{ml} \times$ fasting glucose $(\mathrm{mmol} / \mathrm{l}) / 22.5$ ], in the control group this indicator did not exceed 2.77. Glycated hemoglobin A1c was determined by means of affinity chromatography using microcolumns and standard "Diabetes-test" kits. Regional arterial stiffness was assessed using volumetric sphygmography by means of estimating pulse wave velocity (PWV) and the cardio-ankle vascular index (CAVI). The indices were obtained by means of VaSera-1000 instrument ("Fukuda Denshi", Japan). The method of volumetric sphygmography is presented as a graphic recording of fluctuations of the arterial wall that occur when a pressure wave propagates through the vessels. The following characteristics were registered: PWV in the aorta - the carotid-femoral pulse wave velocity (aortic PWV). The cardio-ankle vascular index (CAVI) was studied on the right (R) and on the left (L) - an index reflecting the real stiffness of the vascular wall of the aorta, femoral and tibial arteries. C-Al and R-Al were defined as indices of growth (augmentation), respectively, on the carotid and brachial arteries, which characterize the reflected wave magnitude. The change in the thickness of the intima-media complex (IMC) of the right and left common carotid arteries (CCA) was studied by means of duplex ultrasonography of carotid arteries on a Mindray DC-6 machine. Statistical processing of the results was carried out by means of methods of variational statistics using software Microsoft 
Excel 2010 packages. Among the processing methods, the Mann-Whitney test and the Fisher's exact test were used. To study the relationship between quantitative characteristics, the Spearman's rank correlation coefficient was applied.

The results of the study and their discussion: The level of systolic blood pressure (ABP) in the group of patients with hypertensive variant of metabolic syndrome was $156.4 \pm 12.5 \mathrm{mmHg}$, diastolic $-97.6 \pm 8.0 \mathrm{mmHg}$. Arterial hypertension $(\mathrm{AH})$ of the 1 st degree was detected in 8 patients (23.5\%), $\mathrm{AH}$ of the 2 nd degree - in $15(44.1 \%), \mathrm{AH}$ of the $3 \mathrm{rd}$ degree - in 11 patients (32.4\%). 9 patients (26.5\%) had macrovascular complications (myocardial infarction, acute cerebrovascular accident) in their medical history. Patients of the 3rd group had a family health history of DM in 19 patients (59.4\%). Type 2 DM was newly diagnosed in 6 patients (18.8\%), impaired glucose tolerance - in 4 patients (12.5\%), impaired fasting glucose - in 3 patients (9.4\%). The glycated hemoglobin A1c was $8.37 \pm 2.26 \%$. The Atherogenic Index in the combined course of COPD and metabolic syndrome was $6.8 \pm 0.14$ (in the control group $3.1 \pm 0.12, p<0.05$ ).

It is interesting to note that patients with COPD showed a greater degree of severity of the main clinical symptoms of the primary disease compared with COPD patients without metabolic syndrome. For example, dyspnoea, productive cough and general weakness worried patients with COPD and metabolic syndrome significantly more, accordingly in 1.4 ( $p<0.05), 1.7(p<0.01)$ and 1.5 times $(p<0.05)$. During the performance of a 6-minute step test, exercise tolerance in the group of patients with comorbid course of COPD and metabolic syndrome was significantly lower by $82.5 \pm 4.8 \mathrm{~m}(\mathrm{p}<0.05)$ than in patients without metabolic syndrome.

In $97.4 \%$ of patients with comorbid course of COPD and metabolic syndrome, a significant increase in the HOMA of insulin resistance (HOMA-IR) to $7.65 \pm 1.24$ was revealed in comparison with the control $(2.3 \pm 1.09, p<0.05)$. It is interesting to note that the level of leptin in the combined course of COPD and metabolic syndrome was 4.6 times higher than in the group of patients with isolated form of COPD. It is interesting to note that in 32 patients (94.1\%) of the 2 nd group there was an increase in endothelin-1, which is considered to be the most powerful vasoconstrictor factor known at the present time. In addition, in 14 patients (41.2\%) of the 2 nd group and in 27 ones (84.4\%) of the 3rd group a significant increase in the level of CRP by $45 \%$ was revealed, which is one of the main markers of activity of inflammation in the vascular wall, that requires consideration during treatment policy. The results that we obtained testified that changes in the level of interleukins in patients the $2 \mathrm{nd}$ and $3 \mathrm{rd}$ were unidirectional in comparison with the 1 st one and they were characterized by a significant increase in the synthesis of proinflammatory cytokines (IL-6, IL-8, TNF- $\alpha$, vascular endothelial growth factor). It is well-known, that PWV is a functional parameter that reflects the elastic properties of a vessel. In our study, the PWV of the aorta in the 2 nd and 3rd groups was $27.5 \%(p<0.001)$ and $19.3 \%$ ( $p<0.01)$, accordingly, significantly higher than the same index in the 1st group, promoting suggestions about a significant lesion of the aorta in a combined course with $\mathrm{AH}$ and diabetic changes. To assess the effect of $\mathrm{AH}$ and proven ischemic heart disease on the hemodynamic characteristics of the aorta, we performed a comparative analysis of the magnitude of PWV in normotonics (group 3) and individuals with $\mathrm{AH}$ in their medical history (group 2) comparable in age. It was revealed that the presence of $\mathrm{AH}$ led to an increase in the local aortic PWV by $27.3 \%$, the combination of AH and IHD by $36.9 \%$ (p $<0.05$ ).

The right IMC index in the 1 st and 2 nd subgroups was $1.0 \pm 0.2 \mathrm{~mm}$ and $1.11 \pm 0.5 \mathrm{~mm}$, respectively. The left IMC index in the 1 st and 2 nd subgroups was $1.0 \pm 0.3 \mathrm{~mm}$ and $1.12 \pm 0.4 \mathrm{~mm}$, respectively. In 12 patients (35.3\%) of the $2 \mathrm{nd}$ subgroup and in 4 patients (12.5\%) of the 3rd group, an increase in the IMC of the CCA was found to be over $1.3 \mathrm{~mm}$. A more pronounced index of the thickness of the intima-media complex of CCA in the presence of AH in comorbidity can be explained by the influence of high ABP on the stretching of the arterial wall, followed by aggravation of this process by atherosclerosis. When performing the correlation analysis, the PWV of the aorta in the 2 nd group was related to the systolic and diastolic ABP indices ( $r=0.95, p<0.01$ and $r=0.98, p<0.01)$, and also the level of leptin $(r=0.87, p<0.05)$, BMI $(r=0.96, p<0.01)$ and CRP $(r=0.91, p<0.01)$, promoting suggestions about the pathogenetic role of subclinical inflammation in atherosclerotic lesions of the aorta in the combined course of COPD and the hypertonic form of the metabolic syndrome, which requires consideration in the therapy of this nosology. The PWV of the aorta in the 3rd group was most closely correlated with the level of glycated hemoglobin A1c $(r=0.83, p<0.05)$ and the triglyceride index $(r$ $=0.88, p<0.05$ ). In addition, the IMC index of carotid artery in patients with comorbid course of COPD and metabolic syndrome is highly correlated with the aortic PWV index $(r=0.92, p<0.01)$, suggesting the simultaneous development of preatheromatous changes in these vessels in patients with comorbid course of COPD. During the performance of the study, the most significant increase in cardio-ankle vascular index (L-CAVI, R-CAVI) parameters was revealed in the 2nd group, respectively, by $23.7 \%(p<0.01)$, and $22.6 \%(p<0,01)$ in comparison with patients of the 1 st group. During the performance of the study in the 3rd group, an increase in indices of L-CAVI and R-CAVI was observed, respectively, by $8.9 \%(p<0.05)$ and $8.2 \%(p<0.05)$ in comparison with patients of the 1 st group. It is interesting to note that exactly in 14 patients (41.2\%) of the 2 nd group with the presence of $\mathrm{AH}$ in their medical history, the absolute values of CAVI of more than 9.5 were recorded, which is indicative of significant structural changes in the vascular wall. It should be noted that the parameters of the cardio-ankle vascular index in conducting the correlation analysis in the 2 nd group were associated with the indices of endothelin-1 $(r=0.89, p<0.01)$, in the 3rd group - with HOMA-IR $(r=0.74, p<0.05)$, as well as with vascular endothelial growth factor $(r=0.87, p<0.01)$. The increase in aortic stiffness in patients with comorbid pathology naturally led to a moderate decrease in the return time of the reflected wave. In this regard, in patients with comorbid course of COPD and metabolic syndrome of the 2nd and 3rd groups, there was a tendency to decrease of C-Al values by $9.2 \%$ and $7.8 \%$, respectively, as well as R-Al values by $8,1 \%$ and $9.3 \%$ respectively. During the study of correlation relationships, we detected negative relationships in the 2 nd and 3 rd groups between the PWV of the aorta and the index characterizing the magnitude of the reflected wave $-\mathrm{C}-\mathrm{Al}$ (respectively, $r=0.92, p$ $<0.01$ and $r=0,88 ; p<0,05)$, which are hemodynamic signs of atherosclerotic vascular lesions in cases of comorbidity. 
Conclusions: Having analyzed the relationship between the state of hemodynamic properties of the aorta and metabolic disorders in COPD, we came to the following conclusions: in general, the aortic stiffness indices closely correlate with the levels of $A B P, B M I$, glycated hemoglobin, endothelin-1, systemic inflammation, which, in our opinion, is a reflection of the unidirectional influence of these factors on the walls of aortic and carotid arteries in this comorbid pathology and the reflection of their important role in the formation of excessive arterial stiffness. Thus, only early diagnosis of hemodynamic abnormalities using the method of volumetric sphygmography and a comprehensive approach for correction of ABP, systemic subclinical inflammation, insulin resistance, lipid and carbohydrate spectra in patients with COPD associated with the metabolic syndrome can promote the improvement of endothelial function, arterial stiffness and consequently, reducing the risk of developing and progressing cardiovascular accidents.

Reference list:

1. Dedov I.I. Morbid obesity. Moscow: Medical Information Agency, 2014. 608 p.

2. Ovcharenko S.I., Galetskaite Ya.K. Roflumilast in Treatment of Patients With Severe Chronic Obstructive Pulmonary Disease Combined With Multimorbid Pathology // Attending Medical Doctor. 2015. № 5. P. 74-78.

3. Pavlenko V.I., Kolosov V.P., Naryshkina S.V. Features of Comorbid Course, Prediction and Treatment of Chronic Obstructive Pulmonary Disease and Coronary Heart Disease. Blagoveschensk, 2014. 260 p.

4. Tanchenko O.A., Naryshkina S.V., Sivyakova O.N. Ursodeoxycholic Acid in Combination Treatement of Patients With Metabolic Syndrome // Russian Journal of Gastroenterology, Hepatology, Coloproctology. 2012. Vol. 22, № 1. P. 82-86.

5. Tanchenko O.A., Naryshkina S.V. Characteristics of Immune Status of Patients With Metabolic Syndrome // Far-Eastern Medical Journal. 2014. № 2. P. 20-23.

6. Chuchalin A.G., Tseimakh I.Ya. Changes in Systemic Inflammatory and Hemostatic Reactions in Patients With Recurrence of Chronic Obstructive Pulmonary Disease With Concomitant Congestive Heart Failure and Obesity // Pulmonology. 2014. № 6. P. 25-32.

7. Wedzicha J.A., Seemungal T.A. COPD exacerbations: defining their cause and prevention // Lancet. 2013. Vol. 370. P. 786-796.

8. Wells C.E., Bacer E.H. Metabolic syndrome and diabetes mellitus in COPD // Eur. Respir. Monograph. 2013. Vol. 59. P. 117-134.

UDC 616.248 - 084:618.2 - 06 DOI 10.22448/AMJ.2017.3.146-148

PECULIARITIES OF RENAL HEMODYNAMICS IN PREGNANT WOMEN WITH BRONCHIAL ASTHMA

Zenkina A.S., Prikhodko O.B., Romantsova E.B., Kostrova I.V.

Amur state medical academy, Blagoveshchensk, Russian Federation

Abstracts. The incidence of bronchial asthma (BA) in the world is from 4 to $10 \%$ of the population; In the Russian Federation, prevalence of adult ranges from 2.2 to $5-7 \%$. At pregnant women, asthma is the most common disease of the pulmonary system, the frequency of diagnosis in the world ranges from 1 to $4 \%$, in Russia - from 0.4 to $1 \%$. The aim of the study was to study the peculiarities of renal hemodynamics in pregnant women with bronchial asthma.

Key words: bronchial asthma, pregnancy, renal hemodynamics

Pregnancy affects the course of asthma differently. Changes in the course of the disease range in a fairly wide range: improvement - in $18-69 \%$ of women, worsening - in $22-44 \%$, the lack of influence of pregnancy on BA is revealed in $27-43 \%$ of cases. This is due, on the one hand, to multidirectional dynamics in patients with varying degrees of severity of asthma (with mild and moderate severity, the deterioration of asthma is observed in $15-22 \%$, improvement in $12-22 \%)$, on the other hand - inadequate diagnosis and not always the right therapy. In practice, asthma is often diagnosed only in the late stages of the disease. In addition, if its onset coincides with the gestational period, the disease may remain unrecognized, since the observed breathing disorders are often attributed to changes due to pregnancy.

Material and methods. Clinical and functional features of the course, pregnancy, delivery and status of newborns in 35 patients with bronchial asthma were analyzed. The observed patients were of mature childbearing age the average age was $25 \pm 1.7$ years. Hereditary complication in allergic diseases was traced in $8(22.8 \%)$ women, while in the BA - in $3(8.6 \%)$, all - on the maternal line. A mild course of asthma (BALT) was noted in $24(68.5 \%)$ patients, moderate (BAST) - in $10(28.8 \%)$, severe (BATT) - in 1 (2.9\%). According to the clinical forms of the disease, the patients are distributed as follows. In 23 (65.7\%) patients, the allergic form of asthma was diagnosed, in $2(5.7 \%)$ was non-allergic, in $10(28.8 \%)$ was mixed. The duration of asthma, on average, was $9.5 \pm 1.3$ years.

Results and discussion. Dynamics of the course of asthma in the dynamics of pregnancy was determined: 22 (62.9\%) had deterioration, with no control in the gestational period, 8 (22.9\%) had no significant dynamics, 5 (14.3\%) had a lighter process. It was noted that the weighting of the symptoms of the disease during pregnancy was in patients with non-allergic and mixed forms of moderate and severe asthma. Among the specific cause-significant factors, polyvalent sensitization to epidermal, medicinal and pollen allergens was more often observed, from non-specific allergens, A.R.W.I., psy- 